\title{
Zur Kinetik der enzymatischen Umwandlung von Citronensäure in cis-Aconitsäure und iso-Citronensäure
}

\author{
Von H. Friedrich-Freksa und C. Martius \\ Aus dem Max-Planck-Institut für Biochemie und dem Physiologisch-chemischen Institut \\ der Universität Tübingen \\ (Z. Naturforschg. 6 b, 296-304 [1951]; eingegangen am 3. Juli 1951)
}

\begin{abstract}
Bei der Entstehung von iso-Citronensäure aus Aconitsäure unter Einwirkung der Aconitase steigt die iso-Citronensäure zunächst weit über den Gleichgewichtswert des Systems Citronensäure-Aconitsäure-iso-Citronensäure an. Die Lösung der reaktionskinetischen Gleichungen ergibt, daß ein Stơf sich auch dann vorübergehend anhäufen könnte, wenn er nicht weiterreagieren, sondern über den Ausgangsstoff in einen dritten Stoff übergehen würde. Nach Durchrechnung verschiedener Möglichkeiten des kinetischen Ablaufs für das Aconitase-System zeigt sich, daß in diesem Fall wahrscheinlich ein einziges Ferment einen Übergang von jeder der drei Säuren zu den beiden anderen bewirkt über eine Ferment-Zwischenverbindung, aus der jede der drei Säuren unmittelbar entstehen kann. Unter dieser Annahme lassen sich die sechs gemessenen Anfangsgeschwindigkeiten und der ebenfalls gemessene vollständige Verlauf zweier Reaktionen in befriedigender Übereinstimmung mit der Erfahrung darstellen.
\end{abstract}

$\mathrm{D}$ urch das Ferment oder Fermentsystem der Aconitase wird in der Zelle Citronensäure in cisAconitsäure und iso-Citronensäure umgewandelt. Im Versuchsansatz mit pflanzlichen oder tierischen Gewebsextrakten stellt sich zwischen diesen drei Säuren ein echtes Gleichgewicht ein, dessen Lage unabhängig davon ist, welche der Säuren als Ausgangsstoff dient. Wird cis-Aconitsäure als Ausgangsstoff gewählt und die Bildung der iso-Citronensäure verfolgt, so zeigt sich, daß die iso-Citronensäure sich bis $\mathrm{zu}$ einem Maximalwert von etwa $30 \%$ anhäuft, um dann erst auf den Gleichgewichtswert von 7\% abzufallen. Dieses Reaktionsverhalten erscheint deswegen überraschend, weil man annehmen sollte, daß die isoCitronensäure auf dem gleichen Wege, auf dem sie entstanden ist, nämlich über die Aconitsäure, wieder verschwindet. Die vorliegenden Untersuchungen nahmen ihren Ausgang von der Frage nach der Deutung dieses Reaktionsverlaufs. Nach der Klärung dieses Problems wurde weiter versucht, den gesamten Reaktionsverlauf des Systems Citronensäure-Aconitsäure-iso-Citronensäure durch eine Theorie der Reaktionskinetik vollständig und quantitativ zu erfassen.

1 B. Natelson, J. K. Luguvoy u. J. B. Pinc u s, J. biol. Chemistry 170, 597 [1947].

2 C. Martius u. F. Ly ne n, Advances in Enzymology Bd. X, S. 193; New York 1950.

3 R a cker, Biochimica et Biophysica Acta 4, 211 [1950].

4 C. M artius u. H. L e o n hardt, Hoppe-Seyler's Z. Physiol. Chem. 278, 208 [1943].

\section{Experimentelle Grundlagen}

Die Citronensäure wurde nach $\mathrm{Natelson}{ }^{1}$ bestimmt, die iso-Citronensäure nach $\mathrm{M}$ artius und $\mathrm{L}$ y $\mathrm{n}$ e $\mathrm{n}^{2}$ unter Verwendung der Werte für die spezifische Drehung des iso-Citronensäure-Molybdat-Komplexes. Die Aconitsäure wurde in den älteren Versuchen, die dieser Arbeit zugrunde liegen, als Differenz der Ausgangsmenge und der Summe von Citronensäure und iso-Citronensäure bestimmt. Im letzten Jahr hatten wir die Möglichkeit, einige direkte Bestimmungen mit dem Beckmann-Spektrophotometer nach $\mathrm{R}$ a c k e $\mathrm{r}^{\mathbf{3}}$ durchzuführen.

Als Grundlage für die theoretische Behandlung muß zunächst die Lage des Gleichgewichts zwischen Citronensäure, iso-Citronensäure und cis-Aconitsäure gegeben sein. Die Gleichgewichtswerte der drei Säuren liegen nach Martius und Le onhardt 4 bei: $89,2 \%$ Citronensäure, $3,1 \%$ cis-Aconitsäure und $7,7 \%$ iso-Citronensäure, wenn als Reaktionsbedingungen etwa eine $m / 10$-Substratkcnzentration, eine Temperatur von $37^{\circ} \mathrm{C}$ und ein $p_{\mathrm{H}}$ von etwa 7,4 gewählt werden. Ferner muß bekannt sein, ob unter den Bedingungen, unter denen der Reaktionsverlauf gemessen wurde, eine Abhängigkeit der Geschwindigkeit des Umsatzes von der Substratkonzentration besteht. Die Konzentration der cis-Aconitsäure als Ausgangsstoff wurde zwischen $m / 2$ und $m / 40$ variiert. Es ergab sich in diesem Bereich keine Abhängigkeit des Umsatzes von der Substratkonzentration. Daraus ist zu schlieBen, daß das Ferment fast vollständig mit Substrat gesättigt ist und daß die Geschwindigkeit des Umsatzes nur von der Fermentkonzentration abhängt.

2. Theoretischer Ansatzinerster Näherung unter Annahme eines Ferments

Unter diesen experimentell festgestellten Voraussetzungen erfordert die Aufstellung der reaktions- 
kinetischen Gleichungen noch eine Annahme darüber, ob die Umwandlung von Citronensäure in Aconitsäure und iso-Citronensäure von einem einzigen Ferment in einem oder in zwei Schritten oder von zwei Fermenten in zwei Schritten katalysiert wird. Wir gehen zunächst von der Annahme aus, daß die Aconitase aus einem Ferment besteht, das in der ersten Stufe Citronensäure in Aconitsäure und in der zweiten Stufe Aconitsäure in iso-Citronensäure umwandelt. Nach dem Vergleich mit der Erfahrung erweist es sich als nötig, anzunehmen, daß ein Ferment in einer Stufe die Umwandlung bewirkt. Schließlich wird noch die Annahme geprüft, daß zwei Fermente in zwei Stufen für die Umwandlung erforderlich sind.

Wenn ein Ferment in zwei Stufen aus Citronensäure iso-Citronensäure entstehen läßt, und die Geschwindigkeit des Umsatzes nur von der Fermentkonzentration abhängt, so lassen sich die folgenden Gleichungen für den Reaktionsverlauf ansetzen:

$$
\begin{gathered}
\frac{\mathrm{d} C}{\mathrm{~d} t}=k_{1} F_{\mathrm{A}}-k_{2} F_{\mathrm{C}}, \\
\frac{\mathrm{d} I}{\mathrm{~d} t}=k_{3} F_{\mathrm{A}}-k_{4} F_{\mathrm{I}} .
\end{gathered}
$$

Hierbei bedeutet $C$ die Konzentration der Citronensäure, $I$ die der iso-Citronensäure, $t$ die Zeit, $F_{\mathrm{A}}$ ist die Konzentration des mit Aconitsäure besetzten Fermentanteils, das Entsprechende gilt für $F_{\mathrm{C}}$ und $F_{\mathrm{I}}$, $k_{1}$ bis $k_{4}$ sind Geschwindigkeitskonstanten. Eine dritte Gleichung für die cis-Aconitsäure erübrigt sich, weil $C+A+I=1$ ist, wenn die Konzentrationen in Molenbrüchen ausgedrückt werden, unter Vernachlässigung des verschwindend kleinen Anteils, der an das Ferment gebunden ist.

Zur Lösung der beiden gekoppelten Differentialgleichungen muß noch der Zusammenhang gegeben sein, der zwischen den relativen Anteilen der Fermentkonzentration und den relativen Anteilen der Substratkonzentration besteht. Bezeichnen wir mit $F$ das freie Ferment, so gilt nach dem Massenwirkungsgesetz:

$C \frac{F}{F_{\mathrm{C}}}=K_{\mathrm{C}}$, entspr. $A \frac{F}{F_{\mathrm{A}}}=K_{\mathrm{A}}$ und $I \frac{F}{F_{\mathrm{I}}}=K_{\mathrm{I}}$.

$K_{\mathrm{C}}, K_{\mathrm{A}}$ und $K_{\mathrm{I}}$ sind die Dissoziationskonstanten der Substrat-Ferment-Verbindungen, die auch MichaelisKonstanten genannt werden. Warburg ${ }^{5}$ fand bei den von ihm untersuchten Fermenten, daß die verschwindenden und die entstehenden Stoffe meistens gleich fest von den Fermentproteinen gebunden wer-

5 O. W a r b u r g, Wasserstoffübertragende Fermente, Berlin 1948. den. Wir machen daher zunächst die einfache Annahme, daß $K_{\mathrm{C}}=K_{\mathrm{A}}=K_{\mathrm{I}}$ ist. Unter dieser Voraussetzung bleibt $F$ zeitlich konstant. Es ergibt sich also, daß die relativen Anteile der Citronensäure, cisAconitsäure und iso-Citronensäure den relativen Anteilen der Fermentkonzentration proportional sind. Durch Umformung erhalten wir die Gleichungen:

$$
\begin{gathered}
\frac{\mathrm{d} C}{\mathrm{~d} t}=a_{\mathrm{c}} A-c_{\mathrm{a}} C, \\
\frac{\mathrm{d} I}{\mathrm{~d} t}=a_{\mathrm{i}} A-i_{\mathrm{a}} I,
\end{gathered}
$$

wobei z. B. $a_{\mathrm{c}}=k_{1} F / K_{\mathrm{A}} . a_{\mathrm{c}}$ bedeutet die Konstante für die Reaktion Aconitsäure $\rightarrow$ Citronensäure, und entsprechend sind die anderen Indices zu verstehen.

Der Reaktionsverlauf ist gegeben, wenn die Konstanten bestimmt werden können und die Anfangsbedingungen bekannt sind. Im Gleichgewicht ist $\mathrm{d} C / \mathrm{d} t$ und $\mathrm{d} I / \mathrm{d} t=0$, daraus folgt:

$$
\frac{a_{\mathrm{c}}}{c_{\mathrm{a}}}=\frac{C_{\infty}}{A_{\infty}}=\frac{89,2}{3,1} \text { und } \frac{a_{\mathrm{i}}}{i_{\mathrm{a}}}=\frac{I_{\infty}}{A_{\infty}}=\frac{7,7}{3,1},
$$

wenn unter $C_{\infty}, I_{\infty}$ und $A_{\infty}$ jeweils die Konzentrationen der Säuren im Gleichgewicht verstanden werden. Nun können die relativen Werte der Konstanten angegeben werden, falls noch das Verhältnis der Geschwindigkeitskonstanten $a_{\mathrm{c}} / a_{\mathrm{i}}$ bekannt ist. Aus der Arbeit von $\mathrm{M}$ artius und $\mathrm{L}$ e o $\mathrm{nh}$ a r d t ergibt sich, daß die Anfangsgeschwindigkeiten der Bildung von Citronensäure und von iso-Citronensäure aus Aconitsäure annähernd gleich sind. Deshalb wird in erster Näherung $a_{\mathrm{c}}=a_{\mathrm{i}}$ gesetzt, womit sich für die relativen Werte der Konstanten die Beziehung:

$$
c_{\mathrm{a}}: a_{\mathrm{c}}\left(=a_{\mathrm{i}}\right): i_{\mathrm{a}}=1: 28,8: 11,6
$$

ergibt. Die absoluten Werte der Konstanten erübrigen sich, weil der Zeitmaßstab unbestimmt bleiben muß, solange die absoluten Fermentkonzentrationen nicht bekannt sind.

Das Einsetzen der erhaltenen Werte in die Gln. (3) und (4), wobei noch $A=1-C-I$ gesetzt wird, führt zu den Gleichungen:

$$
\begin{gathered}
\frac{\mathrm{d} C}{\mathrm{~d} t}=-29,8 C^{-}-28,8 I+28,8, \\
\frac{\mathrm{d} I}{\mathrm{~d} t} \quad-28,8 C-40,4 I+28,8 .
\end{gathered}
$$

Die Integration der beiden gekoppelten Differentialgleichungen nach bekannten Methoden (vgl. z. B. $\mathrm{B}$ a u le ${ }^{6}$ ) gibt:

$$
C-0,893=1,20 A_{1} e^{-5,83 t}+0,83 B_{1} e^{-64,4 t},
$$

6 B. B a u le, Die Mathematik des Naturforschers und Ingenieurs, Bd. IV, S. 340; Hirzel Leipzig 1943. 


$$
I-0,077=A_{1} e^{-5.83 t}+B_{1} e^{-64,4 t},
$$

wobei die beiden Integrationskonstanten $A_{1}$ und $A_{2}$ durch die Anfangsbedingungen festgelegt werden.

Zwei Fälle sollen näher untersucht und mit den experimentellen Erfahrungen verglichen werden.

a) Am Anfang ist nur Aconitsäure vorhanden, dann ist $C=0, I=0$ für $t=0$; für $A_{1}$ ergibt sich dann der Wert 0,41 und für $B_{1}-0,49$. Die Reaktionsgleichung lautet also:

$$
I=0,41 e^{-5,83 t}-0,49 e^{-64,4 t}+0,28 .
$$

b) Am Anfang ist nur Citronensäure vorhanden, dann ist $C=1, I=0$ für $t=0$; für $A_{1}$ ergibt sich dann der Wert $-0,085$ und für $B_{1} 0,008$. Die Reaktionsgleichung lautet also:

$$
I=-0,085 e^{-5,83 t}+0,008 e^{-64,4 t}+0,077 .
$$

\section{Vergleich mit der Erfahrung}

Die Abb. 1 und 2 geben den Vergleich der errechneten Werte mit der Beobachtung wieder. Da der Zeitmaßstab nicht festgelegt ist, wurde er in Abb. 1 so gewählt, daß das Maximum bei beiden Kurven über dem gleichen Abszissenwert zu liegen kommt. Zunächst zeigt der Vergleich der berechneten und beobachteten Kurven, daß der Typus des allgemeinen Reaktionsverlaufs in beiden Fällen eingehalten wird, in Abb. 1 nur in grober Annäherung, in Abb. 2 sogar in ganz guter Übereinstimmung. Wie aus Gl. (10) hervorgeht, fällt der zweite Exponentialausdruck fast weg, so daß der Kurvenverlauf im wesentlichen durch eine einfache Exponentialfunktion wiedergegeben wird, wie es sowohl bei der beobachteten wie bei der berechneten Kurve der Fall ist. Hingegen wird das Maximum in der Abb. 1 durch Überlagerung von zwei Exponentialfunktionen erzielt.

Aus dieser Berechnung folgt eine Deutungsmöglichkeit für die Entstehung des Maximums der iso-Citronensäure, wenn Aconitsäure als Ausgangsstoff gewählt wird. Die größten Geschwindigkeitskonstanten sind $a_{\mathrm{c}}$ und $a_{\mathrm{i}}$, so daß aus Aconitsäure anfangs bis zu etwa $30 \%$ Citronensäure und iso-Citronensäure gebildet werden. Die Rückreaktion iso-Citronensäure $\rightarrow$ Aconitsäure erfolgt langsamer, bei weitem am langsamsten ist aber die Reaktion Citronensäure $\rightarrow$ Aconitsäure, so daß schließlich auch die iso-Citronensäure über die Aconitsäure zur Citronensäure herübergezogen wird.

Diese Möglichkeit ist von einem gewissen theoretischen Interesse, denn im allgemeinen wird der Chemiker aus dem Verschwinden eines intermediär ge- bildeten Stoffes schließen, daß dieser Stoff weiter reagiert. Der hier behandelte Fall zeigt aber, daß ein Stoff auch durch ein Maximum gehen kann, wenn er in einer Nebenreaktion entsteht und auf dem gleichen Wege wieder verschwindet, auf dem er entstanden ist.

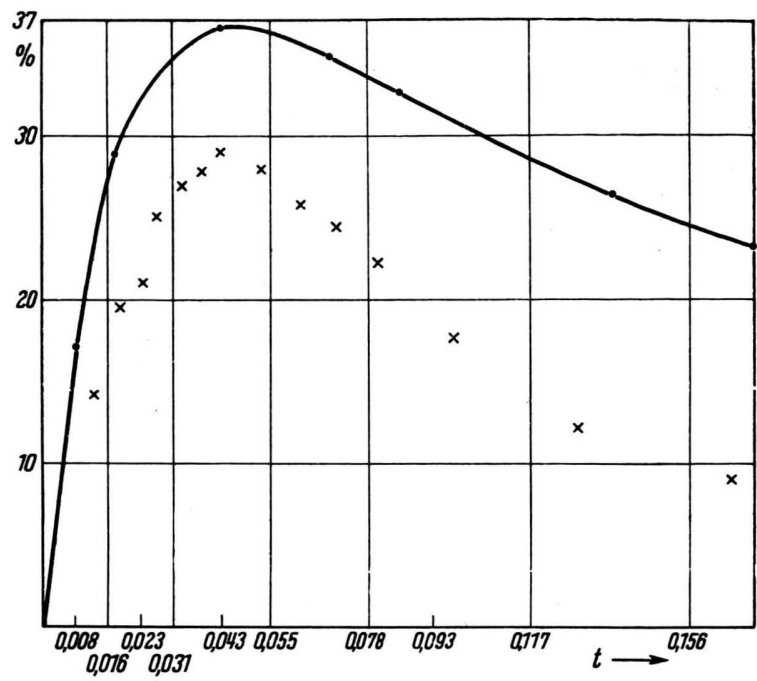

Abb. 1. Bildung von iso-Citronensäure (Ordinate in \%) aus Aconitsäure unter vereinfachten Annahmen und Vergleich mit den beobachteten Werten. - theoretische Kurve, $x$ Meßwerte.

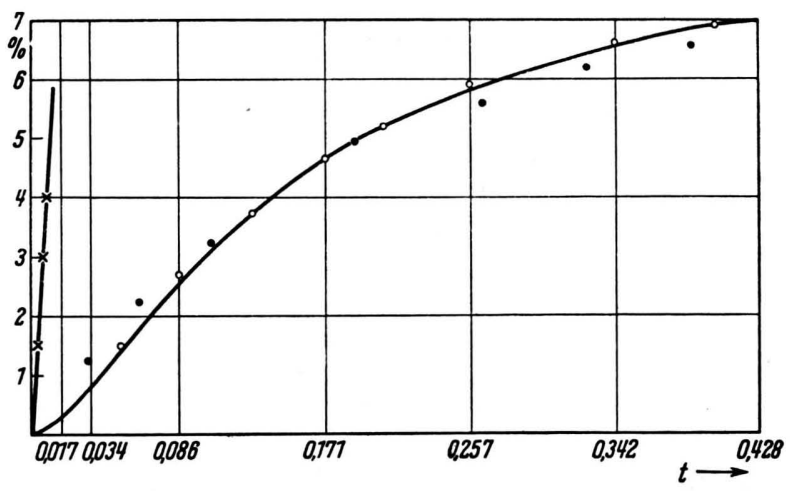

Abb. 2. Bildung von iso-Citronensäure (Ordinate in \%) aus Citronensäure unter vereinfachten Annahmen und Vergleich mit den beobachteten Werten. - theoretische Kurve. x Meßwerte der Versuchsreihe I. ○ Meßwerte der Versuchsreihe II. Die Kurve links oben entspricht dem Zeitmaßstab der Abb. 1.

Zwischen den berechneten und beobachteten Kurven besteht zwar eine gewisse Ähnlichkeit, in quantitativer Hinsicht sind aber die Unterschiede beträchtlich. Das berechnete Maximum liegt in Abb. 1 bei $37 \%$, das beobachtete aber bei $29 \%$. Dieser Unterschied könnte durch eine etwas andere Wahl der Ge- 
schwindigkeitskonstanten verringert werden, aber der wesentlich flachere Abfall nach dem Maximum bei der berechneten Kurve kann auf diese Weise nicht behoben werden. Bei der Abb. 2 scheint zunächst Beobachtung und Berechnung besser übereinzustimmen, aber auch hier besteht eine Abweichung, die sich nur durch eine Änderung der Geschwindigkeitskonstanten nicht beseitigen läßt. Die berechnete Kurve steigt nicht gleich an, sondern sie hat infolge der Überlagerung von zwei Exponentialgliedern eine kleine Anlaufszeit, während die beobachtete Kurve anscheinend ohne eine solche verläuft und auch bei entsprechender Auftragung als Gerade auf halblogarithmischem Papier durch den Nullpunkt geht.

Die stärkste Diskrepanz zwischen Beobachtung und Theorie ergibt sich aber beim Vergleich der anfänglichen Bildungsgeschwindigkeit von iso-Citronensäure aus Citronensäure und Aconitsäure. Theoretisch sollte bei einer Entstehung von 1\% iso-Citronensäure aus Citronensäure als Ausgangsstoff unter den gleichen Bedingungen die Bildung von $35 \%$ iso-Citronensäure aus Aconitsäure in der gleichen Zeit erfolgen. Die wirklich gemessenen Werte entsprechen einem Verhältnis von $1 \%: 3,5 \%$ (vgl. Abb. 3). Die Abb. 1 und 2 stimmen also nur deswegen einigermaßen mit der Beobachtung überein, weil in beiden Fällen für die beobachteten Werte ein anderer Zeitmaßstab gewählt wurde. Im gleichen Zeitmaßstab aufgetragen, würden die ersten Meßpunkte den Kreuzen links auf Abb. 2 entsprechen.

\section{Ausführliche Theoriefür ein Ferment}

Um durch eine Verfeinerung der Theorie eine bessere Übereinstimmung mit der Erfahrung zu erzielen, gingen wir zunächst davon aus, daß die Bildung der iso-Citronensäure aus Citronensäure viel schneller erfolgt, als die vorläufige Theorie fordert. Diese Tatsache, zusammen mit der Beobachtung, daß die gemessene Exponentialfunktion in Abb. 2 ohne feststellbare Latenzzeit vom 0-Punkt an sofort ansteigt, führte uns auf die Vermutung, daß von der Citronensäure auch ein direkter Úbergang in die iso-Citronensäure erfolgen muß, nicht nur auf dem Umwege über die Aconitsäure. Wir stellen uns vor, daß nach der Substratanlagerung an das Ferment eine FermentSubstrat-Zwischenverbindung durchlaufen wird, aus der sowohl Ferment-Aconitsäure wie Ferment-Citronensäure und Ferment-iso-Citronensäure hervorgehen kann. Eine gewisse Stütze für diese Auffassung liefern die Messungen von fünf Anfangsgeschwindig- keiten der Reaktionen unter gleichen Bedingungen, die in Abb. 3 dargestellt sind. Hier zeigt sich, daß auch die Citronensäure mit merklicher Anfangsgeschwindigkeit gebildet wird, wenn iso-Citronensäure als Ausgangsstoff gewählt wird, obgleich die Bildung geringer bleibt als die aus Aconitsäure. Rechnungen, die unter dieser Annahme ausgeführt wurden, ergaben eine etwas bessere Angleichung der Kurve Abb. 1 an die Beobachtung. In dem Bericht von Martius ${ }^{2}$ hat dieses Stadium der Úberlegungen seinen Niederschlag gefunden.

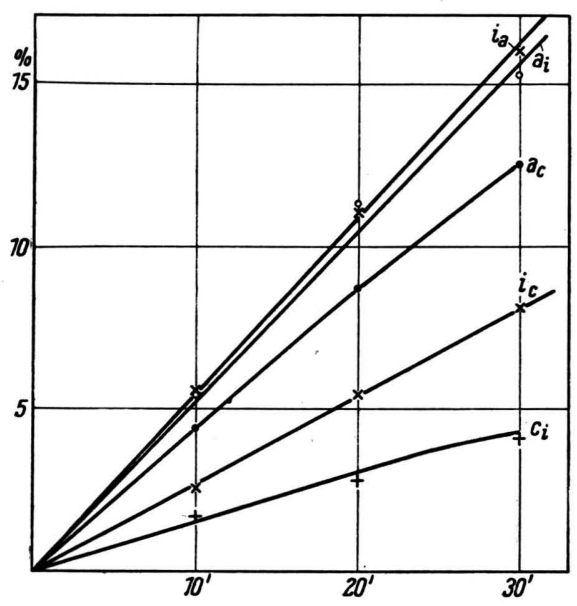

Abb. 3. Anfangsgeschwindigkeiten $i_{\mathrm{a}}, i_{\mathrm{c}}, a_{\mathrm{i}}, a_{\mathrm{c}}, c_{\mathrm{i}}$ bei gleicher Fermentkonzentration gemessen. Substratkonzentration: $m / 25 ; p_{\mathrm{H}} 7,4 ; T 37^{\circ} \mathrm{C}$. Aconitsäure als Differenzbestimmung.

Unter diesen neuen Voraussetzungen lauten die reaktionskinetischen Gleichungen:

$$
\begin{aligned}
& \frac{\mathrm{d} C}{\mathrm{~d} t}=k_{1} F_{\mathrm{A}}+k_{2} F_{\mathrm{I}}-k_{3} F_{\mathrm{C}}, \\
& \frac{\mathrm{d} I}{\mathrm{~d} t}=k_{4} F_{\mathrm{A}}-k_{5} F_{\mathrm{I}}+k_{6} F_{\mathrm{C}} .
\end{aligned}
$$

Setzt man nun wieder die Michaelis-Konstanten $K_{\mathrm{A}}=K_{\mathrm{I}}=K_{\mathrm{C}}$, so ergeben sich die Gleichungen:

$$
\begin{aligned}
& \frac{\mathrm{d} C}{\mathrm{~d} t}=a_{\mathrm{c}} A+i_{\mathrm{c}} I-\left(c_{\mathrm{a}}+c_{\mathrm{i}}\right) C, \\
& \frac{\mathrm{d} I}{\mathrm{~d} t}=a_{\mathrm{i}} A-\left(i_{\mathrm{a}}+i_{\mathrm{c}}\right) I+c_{\mathrm{i}} C .
\end{aligned}
$$

Bei den Koeffizienten der negativen Glieder muß hierbei berücksichtigt werden, daß das Verschwinden auf zwei Wegen erfolgen kann. Versucht man jetzt, diese Gleichungen durch geeignete Wahl der Koeffizienten mit den gemessenen Anfangsgeschwindigkeiten (Abb. 3) und den beobachteten Gleichgewichts- 
werten in Übereinstimmung zu bringen, so zeigt sich, $\mathrm{da} ß$ das auch nicht angenähert möglich ist. Hierbei muß man nämlich noch berücksichtigen, daß durch zwei Gleichgewichtskonstanten, z. B. zwischen Aconitsäure und Citronensäure und zwischen Citronensäure und iso-Citronensäure, die dritte, zwischen Aconitsäure und iso-Citronensäure, bereits gegeben ist. Daher ist durch Wahl von zwei Paaren von Geschwindigkeitskonstanten, z. B. $a_{\mathrm{c}}, c_{\mathrm{a}}$ und $a_{\mathrm{i}}, i_{\mathrm{a}}$, das Verhältnis des letzten Paares $c_{\mathrm{i}} / i_{\mathrm{c}}$ gegeben. Demnach können von den sechs Geschwindigkeitskonstanten nur fünf frei gewählt werden.

Die Unmöglichkeit, Anfangsgeschwindigkeiten und Endwerte mit den Gleichungen in Übereinstimmung

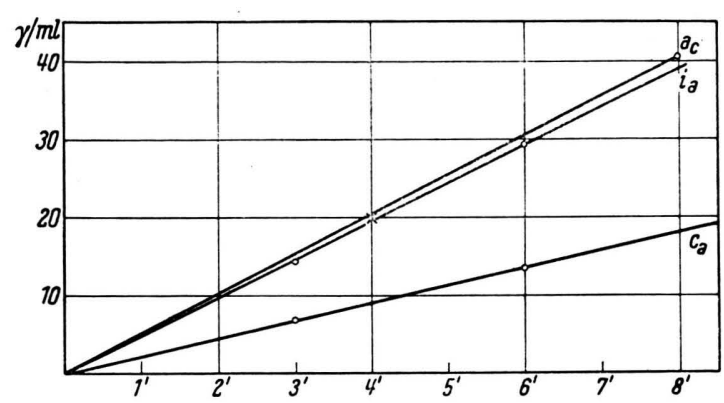

Abb. 4. Gemessene Anfangsgeschwindigkeiten $a_{\mathrm{c}}, i_{\mathrm{a}}, c_{\mathrm{a}}$. Fermentkonzentration in den 3 Ansätzen gleich, aber verschieden von der in Abb. 3. Substratkonzentration $m / 25$; Aconitsäure mit Spektrophotometer bestimmt.

zu bringen, geht nun daraus hervor, daß nach den bisherigen Voraussetzungen durch das Verhältnis der Anfangsgeschwindigkeiten auch die Endwerte bestimmt sind. So würde sich aus dem beobachteten Verhältnis der Geschwindigkeiten $c_{\mathrm{i}} / i_{\mathrm{c}}=1: 1,66$ auch ein entsprechendes Verhältnis von iso-Citronensäure zu Citronensäure im Gleichgewicht ergeben, während der beobachtete Wert ungefähr bei 1:12 liegt. Um eine Übereinstimmung zwischen Anfangsgeschwindigkeiten und Gleichgewichtswerten zu erzielen, muß deshalb die bisher zur Vereinfachung gesetzte Annahme von der Gleichheit der Michaelis-Konstanten fallen. Umgekehrt läßt sich das Verhältnis der Michaelis-Konstanten aus den Abweichungen des Verhältnisses der Anfangsgeschwindigkeiten von den Endwerten berechnen.

Als Anfangsgeschwindigkeiten werden im Anschluß an Abb. 3 folgende Werte angenommen:

$$
a_{\mathrm{i}}=11, i_{\mathrm{a}}=11, i_{\mathrm{c}}=6, c_{\mathrm{i}}=3, a_{\mathrm{c}}=8 .
$$

Der sechste Wert $c_{\mathrm{a}}$ ergibt sich dann daraus zu $c_{\mathrm{a}}=4$. Um hier die Theorie an der Erfahrung zu prüfen, wurde eine Bestimmung des Verhältnisses von $a_{\mathrm{c}} / c_{\mathrm{a}}$ durchgeführt und dabei der Wert 2 gefunden in Úbereinstimmung mit der Erwartung (Abb.4). Etwas abweichend zu Abb. 3 ergab sich hier $a_{\mathrm{c}}=i_{\mathrm{a}}$. In diesem Fall wurde die Bestimmung der Aconitsäure mit dem Beckmann-Spektrophotometer durchgeführt. Aus diesen Geschwindigkeitskonstanten ergibt sich das Verhältnis von $F_{\mathrm{A}}: F_{\mathrm{I}}: F_{\mathrm{C}}$ im Gleichgewicht gleich $1: 1: 2$ nach beistehendem Dreieckschema. Als Gleichgewichtswerte für Citronensäure, Aconitsäure und iso-Citronensäure haben wir nun für die weitere Berechnung die gegen früher etwas veränderten Werte $88 \%, 5 \%$ und $7 \%$ angenommen.

Diese Abänclerung erfolgte, weil nach dem in Abb. 3 wiedergegebenen bis zu 15\% linearen Anstieg der Aconit-

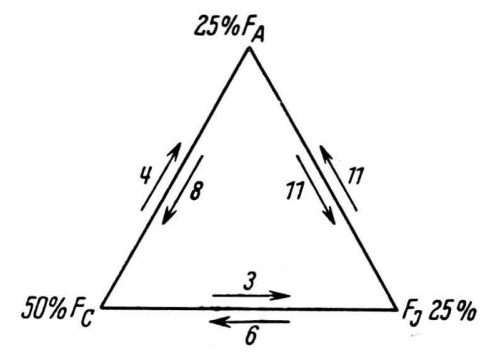

Abb. 5. Schema der Reaktionsgeschwindigkeiten der Ferment-Substrat-Verbindungen unter Angabe der Verhältnisse der Gleichgewichtskonzentrationen der FermentSubstrat-Komplexe.

säure bei der Bildung aus iso-Citronensäure $\left(i_{\mathrm{a}}\right)$ der Gleichgewichtswert der Aconitsäure höher liegen muß als $3,1 \%$. In der schon länger zurückliegenden Arbeit von Martius und Leonhardt 4 wurde der Gleichgewichtswert der Aconitsäure von 3,1\% aus einer Differenzbestimmung durch Einsetzen der Werte von Citronensäure und iso-Citronensäure erhalten, er ist daher weniger gut gesichert als das Ansteigen der Aconitsäure zu höheren Werten. Bei dem Versuch, den Gleichgewichtswert der Aconitsäure neuerdings spektrophotometrisch zu bestimmen, stießen wir auf die Schwierigkeit, daß zur Einstellung des Gleichgewichts hohe Fermentkonzentrationen und damit bei den unreinen Fermentpräparaten auch hohe Konzentrationen an störenden Begleitstoffen erforderlich sind, die sich bisher nicht in einer für die Messung geeigneten Form entfernen ließen.

Wenn nun die Endwerte von $F_{\mathrm{A}}: F_{\mathrm{I}}: F_{\mathrm{C}}$ im Verhältnis 1:1:2 stehen, so müssen die entsprechenden Werte der Michaelis-Konstanten $K_{\mathrm{A}}: K_{\mathrm{I}}: K_{\mathrm{C}}$ etwa das Verhältnis 0,12: 0,15:1 aufweisen, damit die Gleichgewichtswerte von $5 \%: 7 \%: 88 \%$ annähernd herauskommen. Damit sind alle Zahlenwerte gegeben, um die kinetischen Reaktionsgleichungen aufzustellen. In den Gln. (11) und (12) ersetzen wir $F_{\mathrm{A}}, F_{\mathrm{I}}$ und $F_{\mathrm{C}}$ 
jeweils durch $A \cdot F / K_{\mathrm{A}}, I \cdot F / K_{\mathrm{I}}$ und $C \cdot F / K_{\mathrm{C}}$ und erhalten:

$$
\begin{aligned}
& \frac{\mathrm{d} C}{\mathrm{~d} t}=F\left(\frac{k_{1} A}{K_{\mathrm{A}}}+\frac{k_{2} I}{K_{\mathrm{I}}}-\frac{k_{3} C}{K_{\mathrm{C}}}\right), \\
& \frac{\mathrm{d} I}{\mathrm{~d} t}=F\left(\frac{k_{4} A}{K_{\mathrm{A}}}-\frac{k_{5} I}{K_{\mathrm{I}}}+\frac{k_{6} C}{K_{\mathrm{C}}}\right) .
\end{aligned}
$$

Jetzt ist noch $F$ durch $C, I$ und $A$ auszudrücken. Zu diesem Zweck geht man davon aus, daß die gesamte Menge Ferment-Protein konstant ist, und daß ferner die Menge freien Ferment-Proteins gegenüber der Menge gebundenen Proteins zu vernachlässigen ist. Das gibt die Gleichungen:

$$
F_{\mathrm{A}}+F_{\mathrm{I}}+F_{\mathrm{C}}=\text { konstant }
$$

und daraus

$$
A \frac{F}{K_{\mathrm{A}}}+I \frac{F}{K_{\mathrm{I}}}+C \frac{F}{K_{\mathrm{C}}}=\text { konstant }
$$

Da es in diesem Zusammenhang nur auf Verhältniswerte ankommt, kann man die Summe gleich 1 setzen, so daß sich ergibt:

$$
F=\frac{K_{\mathrm{A}} K_{\mathrm{I}} K_{\mathrm{C}}}{K_{\mathrm{I}} K_{\mathrm{C}} A+K_{\mathrm{A}} K_{\mathrm{C}} I+K_{\mathrm{A}} K_{\mathrm{I}} C} .
$$

Einsetzen in 15 und 16 ergibt dann:

$$
\begin{aligned}
& \frac{\mathrm{d} C}{\mathrm{~d} t}=\frac{K_{\mathrm{I}} K_{\mathrm{C}} k_{1} A+K_{\mathrm{A}} K_{\mathrm{C}} k_{2} I-K_{\mathrm{I}} K_{\mathrm{A}} k_{3} C}{K_{\mathrm{I}} K_{\mathrm{C}} A+K_{\mathrm{A}} K_{\mathrm{C}} I+K_{\mathrm{A}} K_{\mathrm{I}} C}, \\
& \frac{\mathrm{d} I}{\mathrm{~d} t}=\frac{K_{\mathrm{I}} K_{\mathrm{C}} k_{4} A-K_{\mathrm{A}} K_{\mathrm{C}} k_{5} I+K_{\mathrm{I}} K_{\mathrm{A}} k_{6} C}{K_{\mathrm{I}} K_{\mathrm{C}} A+K_{\mathrm{A}} K_{\mathrm{C}} I+K_{\mathrm{A}} K_{\mathrm{I}} C} .
\end{aligned}
$$

Die Werte $k_{1}$ bis $k_{6}$ stellen die Anfangsgeschwindigkeiten dar, hierfür wurden wie früher die Werte gewählt:

$k_{1}=a_{\mathrm{c}}=8, \quad k_{2}=i_{\mathrm{c}}=6, \quad k_{3}=c_{\mathrm{a}}+c_{\mathrm{i}}=7$, $k_{4}=a_{\mathrm{i}}=11, \quad k_{5}=i_{\mathrm{a}}+i_{\mathrm{c}}=17, \quad k_{6}=c_{\mathrm{i}}=3$.

Für die Konstanten $K_{\mathrm{A}}, K_{\mathrm{I}}$ und $K_{\mathrm{C}}$ sind die Werte $0,12,0,15$ und 1 vorhin berechnet worden. Einsetzen der Zahlenwerte und Ersatz von $A$ durch $1-C-I$ ergibt schließlich:

$$
\begin{aligned}
\frac{\mathrm{d} C}{\mathrm{~d} t}=\frac{1,2-0,48 I-1,326 C}{0,15-0,03 I-0,132 C}, \\
\frac{\mathrm{d} I}{\mathrm{~d} t}=\frac{1,65-3,69 I-1,596 C}{0,15-0,03 I-0,132 C} .
\end{aligned}
$$

Wir lösen diese beiden simultanen, nicht linearen Differentialgleichungen auf graphischem Wege in folgender Weise. Durch Division der beiden Gleichungen ineinander wird $\mathrm{d} C / \mathrm{d} I$ als Funktion von $C$ und $I$ ausgedrückt. In das C-I-Diagramm (Abb. 6) werden, ausgehend von den drei Anfangsbedingungen die Neigungen eingetragen und auf diese Weise die zusammengehörigen Werte von $C$ und $I$ erhalten. Die Kurven haben bei der Auftragung die angenehme Eigenschaft, daß jede Abweichung von der wahren Richtung sich in der Folge wieder von selbst korrigiert, weil der Endpunkt, auf den alle Kurven zustreben, als Gleichgewichtswert festgelegt ist. Aus diesem $C$-I-Diagramm kann nun für jeden $C$-Wert

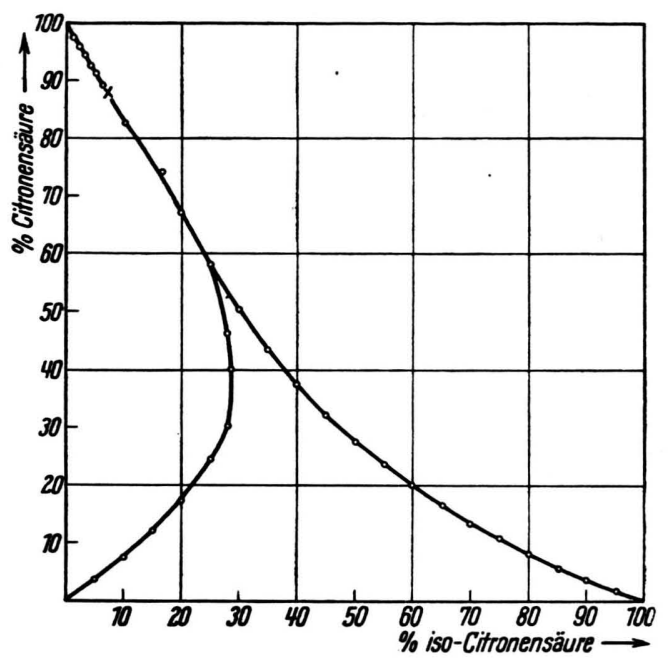

Abb. 6. Citronensäure-iso-Citronensäure-Diagramm nach der verbesserten Ein-Ferment-Theorie. x Gleichgewichtspunkt.

der zugehörige $I$-Wert entnommen werden und in die Gln. (22) und (23) eingesetzt werden. Dann werden die Funktionen $\mathrm{d} t / \mathrm{d} C$ und $\mathrm{d} t / \mathrm{d} I$ graphisch integriert und $t$ jeweils als Funktion von $C$ und $I$ erhalten. Das Ergebnis dieser Rechnung zeigen die Abb. 7, 8, 9 und Tab. 1, welche einen Vergleich gibt der be-

\begin{tabular}{|c|c|c|c|}
\hline & $\begin{array}{c}\text { Beobachtet } \\
\text { \% }\end{array}$ & $\begin{array}{c}\text { Berechnet \% } \\
\text { 1 Ferment }\end{array}$ & $\begin{array}{c}\text { Berechnet \% } \\
\text { 2 Fermente }\end{array}$ \\
\hline$i_{\mathrm{c}}$ & 8 & 10 & 10 \\
$i_{\mathrm{a}}$ & 16 & 14 & 7,5 \\
$a_{\mathrm{c}}$ & 12,5 & 13 & 11,5 \\
$a_{\mathrm{i}}$ & 15,5 & 16 & 10,5 \\
$c_{\mathrm{i}}$ & 4 & 3,5 & 3,4 \\
$c_{\mathrm{a}}$ & - & 3,3 & 3,2 \\
\hline
\end{tabular}

Tab. 1. Beobachtete und berechnete Umsatzwerte an dem Zeitpunkt, an dem sich $8 \%$ Citronensäure aus iso-Citronensäure gebildet haben. 


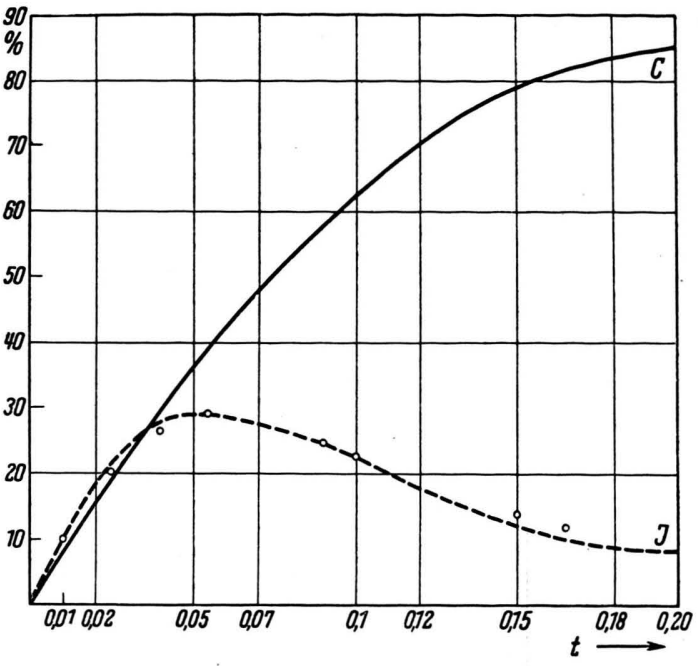

Abb. 7. Bildung von Citronensäure und iso-Citronensäure aus Aconitsäure nach der verbesserten Ein-FermentTheorie.

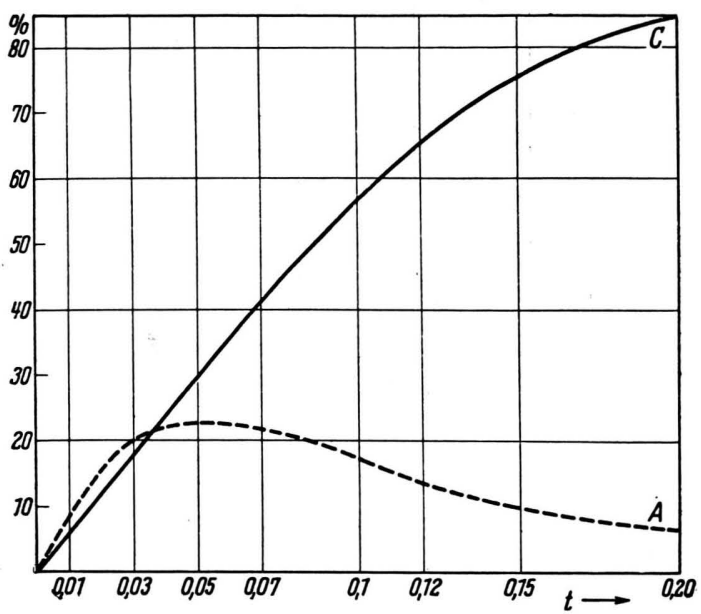

Abb. 8. Bildung von Citronensäure und Aconitsäure aus iso-Citronensäure nach der verbesserten Ein-FermentTheorie.

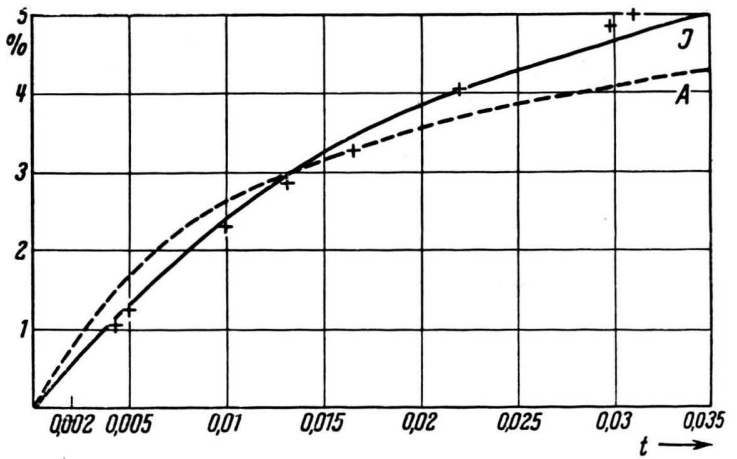

Abb. 9. Bildung von iso-Citronensäure und Aconitsäure aus Citronensäure nach der verbesserten Ein-FermentTheorie. Meßwerte für $I$. obachteten und der berechneten Werte zu dem Zeitpunkt, zu dem sich aus iso-Citronensäure $8 \%$ Citronensäure gebildet haben. Es zeigt sich, daß die maximale Abweichung $20 \%$ beträgt. Über alle Erwartung gut ist die Übereinstimmung bei der Kurve der Entstehung von iso-Citronensäure aus Aconitsäure, hier wird sowohl das Maximum von $29 \%$ wie der allgemeine Kurvenverlauf bis kurz vor dem Ende genau wiedergegeben. Die Abweichung am Schluß ist um so unbedenklicher, als Grund zur Annahme besteht, daß das Ferment in seiner Wirkung zu diesem Zeitpunkt bereits nachläßt.

Zur größeren Sicherheit wurden auch noch mit den alten Gleichgewichtswerten die Berechnungen durchgeführt. Die Übereinstimmung zwischen Beobachtung und Theorie ist dann weniger gut, denn es ergaben sich $36 \%$ für das Maximum von $a_{\mathrm{i}}$ und $15 \%$ für das Maximum von $i_{\mathrm{a}}$.

Eine weitere Prüfungsmöglichkeit der Theorie liefert die experimentelle Bestimmung der MichaelisKonstanten, die von $\mathrm{R} \mathrm{a} \mathrm{c} \mathrm{k} \mathrm{e} \mathrm{r}{ }^{3}$ durchgeführt wurde. Er fand ein Verhältnis der Michaelis-Konstanten von Ferment-iso-Citronensäure zu der von FermentCitronensäure wie 1:3, während wir 1:6 errechneten. Die Michaelis-Konstante der Ferment-Citronensäure ist also auch nach dem Experiment größer, unterscheidet sich aber um einen Faktor 2 von unserem Wert. Hierbei ist zu bedenken, daß die Bestimmung von $\mathrm{Racker^{3 }}$ bei sehr niedrigen Substratkonzentrationen erfolgte und nicht vollständig mit unseren Werten bei hohen Substratkonzentrationen $\mathrm{zu}$ vergleichen ist.

Nach allem kann man sagen, daß die Übereinstimmung zwischen Theorie und Beobachtung unter Berücksichtigung der Ungenauigkeit der Voraussetzung und der Fehlergröße der Beobachtungen befriedigend ist.

\section{Theorie für zwei Fermente}

Aus dem Bisherigen geht hervor, daß durch ein F'erment, das die Umwandlung von Citronensäure in iso-Citronensäure nur über Aconitsäure, also in zwei Stufen, bewirkt, die experimentellen Tatsachen nicht zu erklären sind, weil der bereits am Anfang beträchtliche Wert für die Entstehung von iso-Citronensäure aus Citronensäure bzw. von Citronensäure aus iso-Citronensäure auf diese Weise nicht zustande kommen kann. Durch die Annahme eines Ferments, das auch unmittelbar Citronensäure in iso-Citronensäure und umgekehrt umwandelt, werden die Be- 
obachtungen befriedigend wiedergegeben, und es ist nun die Frage aufzuwerfen, ob nicht eine ebenso gute Deutung auch durch eine Zwei-Ferment-Reaktion möglich ist.

Für zwei Fermente müssen auch zwei Reaktionsstufen angenommen werden, und es scheint nun zunächst, als ob die Schwierigkeit, die für die EinFerment-Reaktion in zwei Stufen in dem Anfangswert der Hin- und Rückreaktion von Citronensäure zu isoCitronensäure sich ergab, auch hier vorhanden sein müsse. Bei genauer Úberlegung zeigt sich aber, daß das nicht der Fall ist. Der Unterschied zur Ein-Ferment-Reaktion in zwei Stufen besteht in folgendem: Wenn etwa aus Citronensäure sich etwas Aconitsäure gebildet hat, so konkurrieren die Substrate Citronensäure und Aconitsäure um das Ferment, und die Umsetzung von Aconitsäure in iso-Citronensäure findet nur in dem kleinen Maße statt, in dem FermentAconitsäure vorhanden ist. Wenn es sich aber um zwei Fermente handelt, so hat das zweite Ferment zunächst als einziges Substrat Aconitsäure, weil es mit Citronensäure nicht reagiert. Der Umsatz von Aconitsäure zu iso-Citronensäure kann gleich in voller Höhe stattfinden, wenn nur die Menge der Aconitsäure so groß geworden ist, daß das Ferment mit Substrat annähernd gesättigt ist.

Die Zwei-Ferment-Theorie läßt sich demnach nicht von vornherein aus der Betrachtung ausschließen, sondern es müssen auch hier die kinetischen Reaktionsgleichungen aufgestellt und mit der Erfahrung verglichen werden. Die Gleichungen lauten hier:

$$
\begin{aligned}
& \frac{\mathrm{d} C}{\mathrm{~d} t}=k_{1} F_{\mathrm{A}}-k_{2} F_{\mathrm{C}}, \\
& \frac{\mathrm{d} I}{\mathrm{~d} t}=k_{3} \Phi_{\mathrm{A}}-k_{4} \Phi_{\mathrm{I}},
\end{aligned}
$$

wobei hier $F_{\mathrm{A}}+F_{\mathrm{C}}$ und $\Phi_{\mathrm{A}}+\Phi_{\mathrm{I}}$ für sich konstant gesetzt werden müssen, und vier Michaelis-Konstanten, die mit $K_{\mathrm{A}}, K_{\mathrm{C}}, K_{\mathrm{A}}^{\prime}, K_{\mathrm{I}}^{\prime}$ bezeichnet werden sollen, auftreten. Unter diesen Umständen ergeben sich in ähnlicher Weise wie im vorigen Abschnitt die Gleichungen:

$$
\begin{gathered}
\frac{\mathrm{d} C}{\mathrm{~d} t}=\frac{k_{1} A-\frac{K_{\mathrm{A}}}{K_{\mathrm{C}}} k_{2} C}{A+\frac{K_{\mathrm{A}}}{K_{\mathrm{C}}} C}, \\
\frac{\mathrm{d} I}{\mathrm{~d} t}=\frac{k_{3} A-\frac{K_{\mathrm{A}}^{\prime}}{K_{\mathrm{I}}^{\prime}} k_{4} I}{A+\frac{K_{\mathrm{A}}^{\prime}}{K_{\mathrm{I}}^{\prime}} I} .
\end{gathered}
$$

Es gilt:

$$
\begin{aligned}
& k_{1}=a_{\mathrm{c}}=1, \\
& k_{2}=c_{\mathrm{a}}+c_{\mathrm{i}}=1, \\
& k_{3}=a_{\mathrm{i}}=1, \\
& k_{4}=i_{\mathrm{a}}+i_{\mathrm{c}}=1,7 .
\end{aligned}
$$

Nach Abb. 4 wurde $a_{\mathrm{c}}$ gleich $i_{\mathrm{a}}$ gesetzt, und da in Abb. $3 i_{\mathrm{a}}$ gleich $a_{\mathrm{i}}$ ist, wurde für $a_{\mathrm{e}}$ und $a_{\mathrm{i}}$ der gleiche Wert 1 angenommen. Da $c_{\mathrm{a}}$ in Abb. 4 den halben Wert hat und $c_{\mathrm{i}}$ ungefähr gleich groß ist, wurde die Summe beider auch gleich 1 gesetzt und $i_{\mathrm{a}}+i_{\mathrm{c}}$ nach Abb. 3 gleich 1,7. (Die entspr. Werte im vorigen $\mathrm{Ab}-$ schnitt sind: $\quad a_{\mathrm{c}}=0,8 ; \quad c_{\mathrm{a}}+c_{\mathrm{i}}=0,7 ; \quad a_{\mathrm{i}}=1,1 ;$ $\left.i_{\mathrm{a}}+i_{\mathrm{c}}=1,7\right)$.

Unter diesen Voraussetzungen und den Gleichgewichtskonstanten von $88 \%, 7 \%$ und $5 \%$, wie früher, ergeben sich für das Verhältnis der MichaelisKonstanten $K_{\mathrm{A}} / K_{\mathrm{C}}$ und $K_{\mathrm{A}}^{\prime} / K_{\mathrm{I}}^{\prime}$ die Werte 0,057 und 0,4. Demnach erhält man die Gleichungen:

$$
\begin{aligned}
\frac{\mathrm{d} C}{\mathrm{~d} t} & =\frac{A-0,057 C}{A+0,057 C}, \\
\frac{\mathrm{d} I}{\mathrm{~d} t} & =\frac{A-0,7 I}{A+0,4 I} .
\end{aligned}
$$

Die Integration nach den gleichen Methoden wie im vorigen Abschnitt führt zu den Werten der Tab. 1, Spalte 4. Die Übereinstimmung ist wesentlich schlechter als mit der Ein-Ferment-Theorie. Die größte Abweichung besteht hier in der nicht wiedergegebenen Kurve der Entstehung der Aconitsäure aus isoCitronensäure, wo als Maximum nur ein Wert von $12 \%$ erreicht wird, während in Abb. 3 noch bei $15 \%$ ein linearer Anstieg erfolgt. Auch die Kurve Aconitsäure zu iso-Citronensäure wird in ihrer Form schlechter wiedergegeben, wenn auch das Maximum von $30 \%$ hier einigermaßen übereinstimmt. Es ist zwar möglich, daß durch eine etwas andere Wahl der Anfangsgeschwindigkeiten sich noch eine bessere Übereinstimmung erzielen läßt, aber solange das nicht gezeigt ist, müssen wir der Ein-Ferment-Theorie den Vorzug geben.

Wir haben auch hier die Rechnung mit den alten Gleichgewichtswerten von 89,2, 7,7 und 3,1\% wiederholt, die Übereinstimmung mit der Beobachtung ist dann noch schlechter.

Es kann auch nicht als Beweis gegen die EinFerment-Theorie angesehen werden, wenn eine Verschiebung der Reaktionsgeschwindigkeiten $i_{\mathrm{a}}$ und $a_{\mathrm{i}}$ einerseits zu $c_{\mathrm{a}}$ und $a_{\mathrm{c}}$ andererseits bei gewissen 
F'ermentpräparaten erfolgt, weil die Möglichkeit besteht, daß an einem Ferment verschiedene Haftgruppen in unterschiedlicher Weise geschädigt werden können. Nur die Isolierung zweier sich als verschieden erweisender Fermentproteine könnte einen einwandfreien Gegenbeweis liefern.

\section{Schlußfolgerungen}

Das Problem der intermediären Anhäufung von iso-Citronensäure, von dem diese Untersuchung ihren Ausgang nahm, ist völlig geklärt. Es ergibt sich zwangsläufig aus dem reaktionskinetischen Ansatz, ob nun der Umsatz durch ein oder durch zwei Fermente bewirkt wird. Der Vergleich der Beobachtung mit der Berechnung macht die Reaktion mit einem Ferment wahrscheinlicher, wobei man jedoch annehmen muß, daß auch ein direkter Übergang von Citronensäure in iso-Citronensäure stattfindet. Nach dieser Auffassung ist die Aconitase also eigentlich eine Citronensäure-Isomerase und die Aconitsäure nur ein Nebenprodukt, das notwendigerweise mit in den Reaktionsverlauf eingeht.

Eine besondere Betrachtung verdient vielleicht noch das Verhältnis der Michaelis-Konstanten von
Citronensäure und iso-Citronensäure bzw. Aconitsäure. Warburg ${ }^{5}$ fand bei den Fermenten des anaeroben Zuckerabbaus mit Ausnahme der Endstufe Gleichheit der Michaelis-Konstanten der jeweils an einem Ferment entstehenden und verschwindenden Stoffe. Es handelt sich hier um Reaktionen, die physiologisch in beiden Richtungen verlaufen. Die Citronensäure wird physiologisch wahrscheinlich nur in Richtung auf die iso-Citronensäure weiter verarbeitet, d.h. sie wird vermutlich im Vergleich zur iso-Citronensäure in einer höheren Konzentration vorliegen, als der Gleichgewichtskonzentration entspricht. Dann erzielt aber der Organismus durch ein Ferment, das eine stärkere Bindung an iso-Citronensäure als an Citronensäure hat, einen schnelleren Umsatz im Vergleich zur Rückreaktion als durch ein Ferment, dessen Michaelis-Konstanten gleich sind; das Verhältnis der Geschwindigkeitskonstanten voir Hin- und Rückreaktion ist durch das Gleichgewichtsverhältnis der Substrate und der Substratfermentverbindungen vollständig bestimmt.

Zu besonderem Dank sind wir Frl. Dr. M. W i e de $\mathrm{m}$ a $\mathrm{n}$ verpflichtet, die die mühevolle rechnerische und graphische Auswertung der Formeln durchführte.

\section{Zur Frage des antibakteriellen Wirkungsmechanismus der Sulfonamide}

VII. Mitteilung: Weitere Untersuchungen über die der Folsäure ähnliche Wachstumswirkung einiger einfacher Pteridinderivate bei Streptococcus faecalis R und ihre Beeinflussung durch Hemmstoffe

Von Rudolf Tschesche, Friedhelm Korte und Ingeborg Korte

Aus der Biochemischen Abteilung des Chemischen Staatsinstitutes der Universität Hamburg

(Z. Naturforschg. 6 b, 304—309 [1951]; eingegangen am 2. Juli 1951)

Die früher von uns als wachstumsfördernd für Strept. faecalis R erkannten Pteridine ließen sich in 2 Gruppen unterteilen, von denen die eine erst bei der Umsetzung mit Zuckern Aktivität erlangt. Die Umsetzung wird auf $N$-Glykosid-Bildung zurückgeführt. Zu dieser Gruppe gehört auch der 2-Amino-6.9-dioxy-pteridinaldehyd-(8), als dessen Vorstufe in Bakterien Iso-xanthopterincarbonsäure wahrscheinlich gemacht wurde. Ihre Wirksamkeit wird durch die entsprechende 8-Essigsäure aufgehoben. Aminopterin vernichtet die Wachstumsaktivität aller als wirksam erkannten Pteridine.

$I^{\prime}$ n der V. Mitteilung ${ }^{1}$ zu diesem Problem hatten wir über die Wachstumswirkung einer Reihe natürlicher und synthetischer Pteridinderivate an Stelle von Pteroyl-glutaminsäure bei Streptococcus faecalis $\mathrm{R}$ berichtet. Es hatte sich damals gezeigt, daß nur solche

1 R. Tschesche, F. Korte u. I. Korte, Z. Naturforschg. $\mathbf{5}$ b, 312 [1950].
Verbindungen dieser Gruppe sich als wirksam erweisen, die eine $\mathrm{NH}_{2}$-Gruppe in 2-Stellung und eine OH-Gruppe in 6-Stellung des Pteridinsystems enthalten. Weitere Variationsmöglichkeiten sind an den C-Atomen 8 und 9 gegeben, doch hatten sich mit Alkyl, Carboxyl oder Hydroxyl substituierte Pteridine an diesen Stellen durchaus nicht alle als wirksam er- 